\title{
EVALUACIÓN PRÁCTICA DE LAS PRUEBAS PARA INVESTIGAR EL FACTOR OVULATORIO COMO PARTE DEL ESTUDIO INICIAL EN LAS PACIENTES CON SUBFERTILIDAD
}

\author{
Pablo Sanhueza R. ${ }^{1,2}$, Jaime Zarhi T. ${ }^{1,2}$, Luis Oliva P. ${ }^{1}$ \\ 1 Departamento de Obstetricia y Ginecología, Escuela de Medicina, Facultad de Ciencias de la Salud, Universidad \\ Andrés Bello. ${ }^{2}$ Unidad de Infertilidad, Servicio de Obstetricia y Ginecología, Hospital El Pino.
}

\section{RESUMEN}

Antecedentes: La subfertilidad corresponde a una condición que afecta entre el 10-17\% de las parejas. Dentro de las causas, los factores anovulatorios corresponden al $27 \%$ de los casos. Las parejas subfértiles deben ser estudiadas con el fin de descartar el factor ovulatorio. No existe consenso en los exámenes que deberían ser realizados en la evaluación inicial del factor ovulatorio. Objetivo: Evaluar críticamente los exámenes disponibles para investigar el factor ovulatorio como parte de la evaluación inicial en las pacientes con subfertilidad. Conclusión: El análisis de la evidencia, establece que la determinación de la progesterona sérica en el día 23 del ciclo menstrual es el examen a realizar en la evaluación inicial de la ovulación en pacientes subfértiles.

\section{PALABRAS CLAVE: Subfertilidad, anovulación, reserva ovárica}

\section{SUMMARY}

Background: Subfertility is a clinical condition which affects between $10-17 \%$ of couples during their reproductive age. Amongst its causes, anovulatory factors are responsible for the $27 \%$ of the cases. In this aspect, subfertile couples should be investigated with the purpose of to rule out anovulation as subfertility cause. However, and in spite of decades of developments, to date there is no consensus on which test should be performed as a part of initial evaluation on these patients. Objective: To critically evaluate the available tests to investigate the anovulatory factor as a component of the initial evaluation on subfertile patients. Conclusion: The analysis of the evidence establishes that the determination of serum progesterone on day 23 of the menstrual cycle, is the test to perform in the initial assessment of ovulation in subfertile patients. 


\section{INTRODUCCIÓN}

La subfertilidad es definida como un año de actividad sexual no protegida sin lograr embarazo. Afecta entre el 10 a $17 \%$ de las parejas durante su edad reproductiva $(1,2)$.

La subfertilidad puede ser dividida en dos tipos: subfertilidad primaria, la cual afecta a aquellas parejas que nunca han logrado un embarazo, y subfertilidad secundaria, la cual afecta a aquellas parejas que han concebido previamente. Considerando las causas de subfertilidad primaria, los factores ovulatorios son responsables del $27 \%$ de los casos, el factor masculino del $25 \%$, los factores tubo-peritoneales del $22 \%$, la endometriosis del $5 \%$, los factores uterinos del $4 \%$, y finalmente en el $17 \%$ de las parejas subfértiles no es posible determinar una causa, siendo estos considerados como inexplicados o de origen desconocido (3).

El $85 \%$ de las parejas lograran un embarazo sin ningún tipo de asistencia después de transcurrido un año de actividad sexual regular y no protegida. Por lo tanto, los test para evaluar la subfertilidad en estos pacientes deberían ser indicados y realizados después de este intervalo. Sin embargo, en aquellas pacientes sobre 35 años, con historia de oligo o amenorrea, cuando su pareja tiene una causa conocida de subfertilidad, o cuando existen antecedentes de endometriosis o se sabe de la presencia de un factor tubo-peritoneal, está indicada una evaluación más temprana y el inicio del tratamiento correspondiente (4).

La evaluación de las parejas subfértiles puede ser difícil debido a que existe una falta de acuerdo con respecto a cuáles pruebas deberían ser realizadas (5). En este sentido, y acorde con los resultados del Capri workshop, las pruebas diagnósticas para el estudio de parejas subfértiles pueden ser separados en tres categorías:

-resultados de pruebas anormales que tienen una establecida correlación con fecundabilidad disminuida: espermiograma, determinación de la permeabilidad tubaria mediante histerosalpingografía o laparoscopia, y evaluación hormonal de la ovulación;

- resultados de pruebas anormales que no están consistentemente correlacionados con fecundabilidad disminuida: test postcoital, hallazgos laparoscópicos en endometriosis leve, histeroscopia, y determinación de anticuerpos anti espermatozoides, entre otros;

- resultados de pruebas anormales que no parecen estar correlacionadas con una fecundabilidad disminuida: determinación del día del ciclo a través de la evaluación histológica del endometrio, evalua- ción de la presencia de varicocele, y faloscopia (5).

La subfertilidad de causa anovulatoria es una condición en la cual puede no haber desarrollo y crecimiento folicular, o de existir, no hay una ruptura del folículo con la subsecuente liberación de un óvulo (5). El embarazo es la evidencia definitiva de que la ovulación ha ocurrido (6), pero no puede ser utilizada como una herramienta clínica. Un método muy útil y que proporciona evidencia directa es el seguimiento folicular desde las etapas iniciales de crecimiento en un ciclo ovárico hasta la ruptura, lo que puede ser realizado a través de la ecografía ginecológica. Sin embargo, es un procedimiento caro y no siempre es posible proponer en todas las pacientes subfértiles como evaluación inicial. Cuatro condiciones han sido descritas como las más frecuentes en mujeres con subfertilidad de causa anovulatoria $(5,7)$, y de las cuales se pueden extrapolar los exámenes a realizar dependiendo de la causa que se sospeche. Estas son las definidas por la World Health Organization (WHO):

- WHO grupo I: no hay evidencia de producción endógena de estrógenos, existen niveles de hormona folículo estimulante (FSH) normales o bajos, niveles de prolactina normales, y no hay evidencia de una lesión en la región hipotálamo pituitaria;

- WHO grupo II: hay evidencia de una producción normal de estrógenos y $\mathrm{FSH}$, y niveles de prolactina normales. En este grupo el síndrome de ovario poliquístico (PCOS) es la principal y mayor causa; - WHO grupo III, la cual involucra niveles de FSH elevados indicando falla a nivel ovárica.

Un grupo final incluye aquellas pacientes con niveles elevados de prolactina. Considerando PCOS, esta es una importante causa de anovulación en pacientes subfértiles, por lo que aquellas mujeres que durante el examen clínico cumplan los criterios deben ser evaluadas con una ecografía transvaginal y test bioquímicos, de acuerdo a los llamados criterios de Rotterdam (8).

Sin embargo, y a pesar de estas consideraciones hoy en día no existe acuerdo en cuál o cuáles exámenes deberían ser realizados como parte de la investigación inicial del factor ovulatorio en pacientes con subfertilidad. Es por ello que hemos querido realizar una evaluación práctica a los test disponibles utilizados como parte de la investigación inicial, para descartar el factor ovulatorio como causa de esta.

\section{Exámenes para descartar el factor ovulatorio en pacientes con subfertilidad.}

A. Evaluación clínica. Todas las parejas subfértiles deben ser clínicamente evaluadas. Con respecto al 
estado ovulatorio debe siempre ser realizados una completa anamnesis y examen físico. En este sentido, pareciera ser que la presencia de ciclos espontáneos y regulares es un muy buen predictor de ovulación ya que se ha demostrado que cuando estos están presentes, $97 \%$ de las mujeres presentan ovulaciones normales (6). Con respecto a la edad, esta tiene una muy estrecha relación con el número de folículos presentes y la calidad de estos. Este hecho, parece estar mas relacionado aun con el número de folículos antrales pequeños entre 2 a $6 \mathrm{~mm}$ (9), los cuales pareciera ser tienen mayor relación con el estado folicular. Otros métodos clínicos indirectos son la determinación de la temperatura basal corporal y su variación durante el ciclo menstrual, y el análisis de las características del moco cervical $(6,10)$. Considerando las variaciones en la temperatura corporal, ha sido demostrado que tiene una sensibilidad de un $77 \%$ y una precisión del $74 \%$ en la detección de ovulación, lo que le resta confiabilidad como prueba (6). Además, la medición de la temperatura corporal basal puede ser afectada por otros factores. Estas observaciones podrían ser igual de válidas para las características del moco cervical. Con respecto a la historia menstrual en la evaluación de la subfertilidad, este desorden puede significar la presencia de otras condiciones endocrinológicas, como PCOS, hiperprolactinemia, amenorrea hipotalámica, falla ovárica e hipotiroidismo, entre otras enfermedades (7), por lo que los hallazgos clínicos de la historia menstrual además de imprecisos, deben ser siempre correlacionados en un esfuerzo para descartar estas patologías.

Por lo tanto, a pesar de que la historia clínica y examen físico tienen un importante rol en la evaluación inicial de estas pacientes, la determinación del estado ovulatorio a través de estos es limitada, y la sospecha clínica de la anovulación siempre debe ser confirmada por exámenes adicionales.

B. Determinación de los niveles de hormona luteinizante $(L H)$ y de los niveles de progesterona séri$c a$. La determinación de LH en sangre y los niveles de progesterona sérica en la mitad de la fase lútea o en el día $23^{\circ}$ del ciclo, son considerados como métodos indirectos para evaluar la ovulación en pacientes subfértiles $(1,6)$. La determinación de LH tiene una alta sensibilidad y una alta precisión para predecir ovulación (100\% y $97 \%$ respectivamente) (6). Por otro lado, la determinación de progesterona sérica en el día $23^{\circ}$ del ciclo menstrual ha demostrado también una alta sensibilidad y precisión (80 y $79 \%$ respectivamente) en la predicción de ovulación (6). Sin embargo, la especificidad de la progesterona sérica es largamente superior cuando esta es comparada con LH (71 y $25 \%$ respectivamente) en la detección de ovulación (6).

Por lo tanto, la evidencia demuestra que la determinación de la progesterona sérica es más confiable en la detección de ovulación, siendo una útil herramienta diagnóstica en la evaluación inicial para descartar la anovulación como causa de subfertilidad. Además, la determinación de los niveles de progesterona sérica pueden ser realizados en días específicos de la fase lutea del ciclo menstrual, a diferencia de la determinación de LH, la cual puede mostrar resultados falsos negativos debido a las variables intrínsecas de la fase folicular.

C. Determinación de los niveles de prolactina y evaluación de la función tiroidea. La determinación de los niveles de prolactina en pacientes con subfertilidad debería ser reservado para aquellas mujeres que presenten síntomas, tales como galactorrea o una alteración de la ciclicidad menstrual normal, debido a que no ha sido posible demostrar una significativa relación entre los niveles de prolactina, los niveles de progesterona sérica y las tasas de concepción acumulada en aquellas mujeres en las cuales se ha demostrado que ovulan (11).

Con respecto a la evaluación de la función tiroidea, es sabido que la disfunción de la glándula tiroides puede producir anovulación, y por lo tanto puede ser una causa de subfertilidad por causa anovulatoria. Sin embargo, sólo un $5,1 \%$ de las mujeres subfértiles presentan una función tiroidea anormal, cifra que se puede elevar hasta un 11,3\% de hipotiroidismo subclínico en aquellas pacientes en las cuales se ha demostrado anovulación $(12,13,14)$.

Por lo tanto, a pesar de que la evaluación de los niveles de prolactina y la evaluación de la función tiroidea podrían demostrar un estado subclínico, estos exámenes deberían ser reservados a aquellas pacientes con manifestaciones clínicas de las condiciones, y no ofrecidos como parte de la evaluación inicial para descartar la causa anovulatoria en las pacientes con subfertilidad.

D. Seguimiento folicular por medio de ecografías transvaginales seriadas. La evaluación ultrasonográfica transvaginal seriada de los folículos en crecimiento, la siguiente formación de un cuerpo lúteo y su visualización, es considerado como el procedimiento "gold standard" para evaluar la ovulación y definir el estado ovulatorio $(6,10)$. La ecografía transvaginal permite ver el número y tamaño de los folículos en crecimiento, evidenciando el crecimiento folicular, la ruptura del folículo pre ovulatorio, la subsiguiente aparición de ecos internos (lo que sugiere la formación del cuerpo lúteo en el espacio donde antes estaba la imagen folicular) y la presencia de líquido libre en el fondo de saco recto 
vaginal, lo que demuestra ovulación (4). Además, la ecografía transvaginal ofrece ventajas adicionales a la sola determinación del estado ovulatorio, como lo es el papel fundamental que juega en la diagnostico de PCOS, donde si bien es posible obtener una imagen ovárica normal, forma parte de la evaluación diagnóstica de estas pacientes de acuerdo a los criterios de Rotterdam (8).

Sin embargo, debido a que las pacientes que presentan amenorreas prolongadas muy probablemente no ovulan, es que en ellas el seguimiento folicular ecográfico tiene utilidad limitada como prueba exclusiva para determinar la presencia o ausencia de ovulación. Pese a ser considerado el "gold standard" para establecer el estado ovulatorio, es difícil de proponerlo como examen de primera línea en estas pacientes, debido a que el seguimiento folicular involucra ecografías seriadas lo que encarece su costo.

D. Biopsia endometrial. La evaluación histológica del endometrio puede ser realizada para demostrar los cambios secretorios durante el desarrollo endometrial, y por lo tanto definir el estado ovulatorio, el cual seria confirmado al demostrar los cambios propios producidos en la fase lútea. Sin embargo, la biopsia endometrial tiene varias desventajas, debido a que el realizar el examen no se relaciona con mejoras en la fertilidad de las pacientes (15), y su precisión ha sido cuestionada y puesta en duda $(10,16)$. Además, el defecto de fase lútea, que es un desorden endocrinológico considerado como una de las principales contribuciones diagnósticas del examen, ha sido puesto en duda que sea causa de subfertilidad $(4,17)$.

Por lo tanto, a pesar de que la biopsia endometrial podría ser considerada como un examen útil para determinar si hubo ovulación, es difícil proponerlo como método para evaluar pacientes subfértiles, dado que no solo es caro e invasivo, sino que además no existe consenso con respecto a su real eficacia en la detección de ovulación (5).

E. Exámenes para determinar la reserva ovárica. La reserva ovárica es definida como el número y calidad de los folículos remanentes en cualquier momento, y puede ser considerado como un indicador del potencial reproductivo de la paciente $(4,18)$. En pacientes subfértiles, la edad juega un papel fundamental en la declinación de la capacidad reproductiva, produciéndose variaciones substanciales entre las mujeres (18). Por lo tanto, las pruebas para determinar la reserva ovárica podrían ser útiles no solo para evaluar las causas de subfertilidad en estas pacientes, sino que también para identificar aquellas mujeres con un alto riesgo de producir una pobre respuesta a la estimulación ovárica (18).
A pesar de que las pruebas para determinar la reserva ovárica son largamente indicadas, el detalle y análisis independiente de estas escapa del objetivo de esta revisión.

La determinación basal de FSH (19), la determinación de la hormona anti Mulleriana (20), inibina B (21), estradiol basal (22), recuento de folículos antrales (23), la determinación del volumen ovárico (21), el test de clomifeno (24), la administración exógena de $\mathrm{FSH}$ (23), el test de estimulación con agonistas del factor liberador de gonadotrofinas (25) y la biopsia ovárica (26), han logrado demostrar tener una buena correlación en la determinación del estado folicular y son utilizados como herramientas predictivas en pacientes subfértiles.

Un reciente meta análisis demostró que las pruebas para determinar la reserva ovárica tienen sólo una modesta capacidad predictiva, y que estas no son adecuadas ni relevantes en el uso clínico (18). Además, los exámenes para determinar la reserva ovárica no solamente son usados como prueba diagnóstica, sino también predictivos. Por lo tanto, un falso positivo, podría excluir pacientes de tratamientos de subfertilidad futuros, por lo que estos debieran ser considerados principalmente como prueba de tamizaje (18). Este punto ha sido considerado también en un reciente estudio donde todos los test para determinar la reserva ovárica fueron independientemente analizados, confirmando que no solo existe una variabilidad entre cada ciclo menstrual si no que también han demostrado tener un rango amplio de sensibilidades, especificidades y razones de probabilidad. Más aún, ninguno de los exámenes disponibles o combinación de ellos, ha sido capaz de predecir embarazo o la tasa de recién nacidos con alta eficacia $(27,28)$. Claramente un determinado resultado no confirma necesariamente si una paciente ovula, salvo que demuestre ausencia de folículos o niveles hormonales en rango de menopausia, como es el caso de la determinación basal de FSH. Puede ser argumentado que estos exámenes serían útiles solo con el propósito de ayudar a las parejas subfértiles, concernientes a la potencial pérdida de la capacidad reproductiva, y como evaluación en aquellas pacientes que serán tratadas con quimioterapia, en un esfuerzo para preservar su fertilidad (28).

Concluyendo este punto y a pesar de que la determinación de la reserva ovárica es un asunto controversial, es posible que no debieran ser indicados como parte de la evaluación inicial en pacientes subfértiles. Sin embargo, la determinación de los niveles de $\mathrm{FSH}$ y estradiol en el día tres del ciclo menstrual son exámenes económicos, ampliamente disponibles, de fácil interpretación y útiles en la 
evaluación inicial del estado ovulatorio, permitiendo descartar falla ovárica y ayudando a clasificar a las mujeres subfértiles dentro de los grupos anovulatorios propuestos por la WHO.

\section{COMENTARIOS}

Determinar la causa de subfertilidad en parejas subfértiles es fundamental, debido a que gran parte de los tratamientos disponibles de primera línea se basan principalmente en tratar de corregir la etiología de base, y por lo tanto descartar la anovulación como causa de subfertilidad juega un rol clave. De acuerdo a estadísticas internacionales, la anovulación es causa de subfertilidad en aproximadamente el $27 \%$ de las parejas subfértiles.

En nuestro medio no existen estadísticas actualizadas ni trabajos prospectivos que demuestren cifras confiables de las reales causas de subfertilidad, debiéndose asumir estadísticas extranjeras. Este punto es importante, ya que la orientación y elección de los exámenes a realizar se debería basar en factores clínicos y epidemiológicos. Afortunadamente, la evaluación inicial de las parejas subfértiles puede ser realizada con relativamente pocos exámenes, tendientes a descartar básicamente factores ovulatorios, tubo peritoneales y masculinos.

Con respecto al estudio de la anovulación como causa de subfertilidad, diversos exámenes han sido desarrollados y realizados por décadas, los que van desde la simple anamnesis y examen físico, hasta la invasiva biopsia endometrial, pasando por diversos exámenes para determinar la reserva ovárica. El análisis crítico de ellos, establece que la evaluación del estado ovulatorio mediante la determinación de progesterona sérica en la mitad de la fase lútea además de sencillo, económico y no invasivo, ofrece alta sensibilidad y especificidad. Si bien el seguimiento folicular ecográfico es considerado el "gold standard" en la determinación de la ovulación, es difícil de proponerlo como método inicial dada sus desventajas ya expuestas.

Respecto a la determinación de prolactina y el estudio de la función tiroidea, estos deben ser reservados para aquellas pacientes que presenten manifestaciones clínicas de la enfermedad.

Los exámenes para determinar la reserva ovárica, deben ser ofrecidos como exámenes predictivos y para consejo reproductivo, pero no como parte de la evaluación inicial en la determinación del estado ovulatorio en estas pacientes.

\section{CONCLUSIÓN}

El análisis de la evidencia, establece que la determinación de la progesterona sérica en el día 23 del ciclo menstrual es el examen a realizar para evaluar la ovulación en pacientes subfértiles. De esta forma se podrá determinar con alta sensibilidad y especificidad si existe un factor ovulatorio causal. Una vez establecido y habiéndose determinado otras posibles causas de subfertilidad dentro de la evaluación inicial, el enfoque terapéutico debe ir dirigido a corregir esta causa, que dependerá principalmente del factor causal de la anovulación.

\section{BIBLIOGRAFÍA}

1. Hull M, Glazener C, Kelly N, Conway D, Foster P, Hinton R, et al. Population study of causes, treatment, and outcome of infertility Br Med J 1985;291:1693-7.

2. Mosher W, Pratt W. Fecundity and infertility in the United States: incidence and trends. Fertil Steril 1991;56:192-3.

3. Collins J. Unexplained infertility. In: Keye W, Chang R, Rebar R, Soules M., (eds). Infertility: Evaluation and treatment. Philadelphia, WB Saunders. 1995.

4. The Practice Committee of the American Society for Reproductive Medicine. Optimal evaluation of the infertile female. Fertil Steril 2006;86:264-7.

5. The ESHRE Capri Workshop. Infertility revisited: the state of the art today and tomorrow. The ESHRE Capri Workshop. European Society for Human Reproduction and Embryology. Hum Reprod 1996;11:1779-807.

6. Guermandi E, Vegetti W, Bianchi M, Uglietti A, Ragni $G$, Crosignani P. Reliability of ovulation tests in infertile women. Obstet Gynecol 2001;97:92-6.

7. The Practice Committee of the American Society for Reproductive Medicine. Current evaluation of amenorrhea. Fertil Steril 2006;86:148-55.

8. The Rotterdam ESHRE/ASRM-sponsored PCOS consensus workshop group. Revised 2003 consensus on diagnostic criteria and longterm health risks related to polycystic ovary syndrome (PCOS). Hum Reprod 2004;19:41-7.

9. Muttukrishna S, McGarrigle H, Wakim R, Khadum I, Ranieri D, Serhal P. Antral follicle count, anti-mullerian hormone and inhibin $\mathrm{B}$ : predictors of ovarian response in assisted reproductive technology? $\mathrm{Br} \mathrm{J}$ Obstet Gynaecol 2005;112:1384-90.

10. Shoupe D, Mishell D, Lacarra M, Lobo R, Horenstein J, d'Ablaing G, Moyer D. Correlation of endometrial maturation with four methods of estimating day of ovulation. Obstet Gynecol 1989;73:88-92.

11. Stratford G, Barth J, Rutherford A, Balen A. Plasma prolactine measurement is not indicated in women in the routine investigation of uncomplicated infertility. Hum Fertil (Camb) 1999;2:70-1.

12. Strickland D, Whitted W, Wians F. Screening infertile women for subclinical hypothyroidism. Am J Obstet Gynecol 1999;163:262-3.

13. Shalev E, Eliyahu S, Ziv M, Ben M. Routine thyroid function tests in infertile women: are they necessary? Am J Obstet Gynecol 1994; 171:1191-2. 
14. Stratford G, Barth J, Rutherford A, Balen A. Value of thyroid function tests in routine screening of women investigated for infertility. Hum Fertil (Camb) 2000;3:203-6.

15. Wentz A, Kossoy L, Parker R. The impact of luteal phase inadequacy in an infertile population. Am J Obstet Gynecol 1990;162:937-43.

16. Li TC, Dockery P, Rogers A, Cooke I. How precise is histologic dating of endometrium using the standard dating criteria? Fertil Steril 1989;51:759-63.

17. Batista M, Cartledge T, Merino M, Axiotis C, Platia M, Merriam G, et al. Midluteal phase endometrial biopsy does not accurately predict luteal function. Fertil Steril 1993;59:294-300.

18. Broekmans F, Kwee J, Hendriks D, Mol B, Lambalk C. A systematic review of tests predicting ovarian reserve and IVF outcome. Hum Reprod Update 2006;12:685718.

19. Akande V, Keay S, Hunt L, Mathur R, Jenkins J, Cahill D. The practical implications of a raised serum FSH and age on the risk of IVF treatment cancellation because of a poor ovarian response. J Assist Reprod Genet 2004;21:257-62.

20. Muttukrishna $S$, Suharjono $H$, McGarrigle $H$, Sathanandan M. Inhibin B and anti-Mullerian hormone: markers of ovarian response in IVF/ICSI patients? $\mathrm{Br} \mathrm{J}$ Obstet Gynaecol 2004;111:1248-53.

21. Erdem M, Erdem A, Gursoy R, Biberoglu K. Comparison of basal and clomiphene citrate induced FSH and inhibin B, ovarian volume and antral follicle counts as ovarian reserve tests and predictors of poor ovarian response in IVF. J Assist Reprod Genet 2004;21:37-
45.

22. Ranieri D, Phophong P, Khadum I, Meo F, Davis C, Serhal P. Simultaneous evaluation of basal FSH and oestradiol response to $\mathrm{GnRH}$ analogue (F-G-test) allows effective drug regimen selection for IVF. Hum Reprod 2001;16:673-5.

23. Yong P, Baird D, Thong K, McNeilly A, Anderson R. Prospective analysis of the relationships between the ovarian follicle cohort and basal FSH concentration, the inhibin response to exogenous FSH and ovarian follicle number at different stages of the normal menstrual cycle and after pituitary down-regulation. Hum Reprod 2003;18: 35-44.

24. Hendriks D, Broekmans F, Bancsi L, de Jong F, Looman C, Te Velde E. Repeated clomiphene citrate challenge testing in the prediction of outcome in IVF: a comparison with basal markers for ovarian reserve. Hum Reprod 2005; 20:163-9.

25. Padilla S, Bayati J, Garcia J. Prognostic value of the early serum estradiol response to leuprolide acetate in in vitro fertilization. Fertil Steril 1990;53:288-94.

26. Lass A. Assessment of ovarian reserve: is there still a role for ovarian biopsy in the light of new data? Hum Reprod 2004;19:467-9.

27. Gulekli B, Bulbul Y, Onvural A, Yorukoglu K, Posaci C, Demir N, Erten O. Accuracy of ovarian reserve tests. Hum Reprod 1999;14:2822-6.

28. Maheshwari A, Fowler P, Bhattacharya S. Assessment of ovarian reserve-should we perform tests of ovarian reserve routinely. Hum Reprod 2006;21:2729-35. 